\title{
Parametric Response Mapping of Apparent Diffusion Coefficient as an Imaging Biomarker to Distinguish Pseudoprogression from True Tumor Progression in Peptide-Based Vaccine Therapy for Pediatric Diffuse Intrinsic Pontine Glioma
}

\author{
R. Ceschin, B.F. Kurland, S.R. Abberbock, B.M. Ellingson, H. Okada, R.I. Jakacki, I.F. Pollack, and A. Panigrahy
}

O- $\equiv$

\begin{abstract}
BACKGROUND AND PURPOSE: Immune response to cancer therapy may result in pseudoprogression, which can only be identified retrospectively and may disrupt an effective therapy. This study assesses whether serial parametric response mapping (a voxel-by-voxel method of image analysis also known as functional diffusion mapping) analysis of ADC measurements following peptide-based vaccination may help prospectively distinguish progression from pseudoprogression in pediatric patients with diffuse intrinsic pontine gliomas.
\end{abstract}

MATERIALS AND METHODS: From 2009 to 2012, 21 children, 4-18 years of age, with diffuse intrinsic pontine gliomas were enrolled in a serial peptide-based vaccination protocol following radiation therapy. DWI was acquired before immunotherapy and at 6-week intervals during vaccine treatment. Pseudoprogression was identified retrospectively on the basis of clinical and radiographic findings, excluding DWI. Parametric response mapping was used to analyze 96 scans, comparing ADC measures at multiple time points (from the first vaccine to up to 12 weeks after the vaccine was halted) with prevaccine baseline values. Log-transformed fractional increased ADC, fractional decreased $A D C$, and parametric response mapping ratio (fractional increased ADC/fractional decreased ADC) were compared between patients with and without pseudoprogression, by using generalized estimating equations with inverse weighting by cluster size.

RESULTS: Median survival was 13.1 months from diagnosis (range, 6.4-24.9 months). Four of 21 children (19\%) were assessed as experiencing pseudoprogression. Patients with pseudoprogression had higher fitted average log-transformed parametric response mapping ratios $(P=$ .01) and fractional decreased ADCs $(P=.0004)$, compared with patients without pseudoprogression.

CONCLUSIONS: Serial parametric response mapping of ADC, performed at multiple time points of therapy, may distinguish pseudoprogression from true progression in patients with diffuse intrinsic pontine gliomas treated with peptide-based vaccination.

ABBREVIATIONS: DIPG = diffuse intrinsic pontine gliomas; $f \mathrm{ADC}=$ fractional decreased $A D C ;$ f $A D C=$ fractional increased $A D C ;$ PRM $=$ parametric response mapping; PRMratio $=$ ratio of fIADC to fdADC; WGEE = weighted generalized estimating equations

$D^{\text {i }}$ iffuse intrinsic pontine gliomas (DIPG) are highly malignant brain stem tumors affecting primarily children. ${ }^{1}$ One-year progression-free survival is $<25 \%$, with a median overall survival

Received February 24, 2015; accepted after revision April 5

From the Departments of Radiology (R.C., A.P.), Pediatrics (R.I.J.), Surgery (H.O.), Neurosurgery (H.O., I.F.P.), Immunology (H.O.), and Biomedical Informatics (R.C., A.P.), University of Pittsburgh School of Medicine, Pittsburgh, Pennsylvania; Departments of Radiology (R.C., A.P.), Pediatrics (R.I..), and Neurosurgery (I.F.P.), Children's Hospital of Pittsburgh, University of Pittsburgh Cancer Institute (B.F.K., S.R.A., H.O., R.I.J., I.F.P., A.P.), and Department of Biostatistics, Graduate School of Public Health (B.F.K.), University of Pittsburgh, Pittsburgh, Pennsylvania; and Department of Radiological Sciences (B.M.E.), University of California, Los Angeles, Los Angeles, California.

This clinical trial was supported by National Institutes of Health grants R21CA149872 and P01NS40923 and the University of Pittsburgh Cancer Institute Immunological Monitoring Core and Biostatistics shared resource facility, supported in part by National Institutes of Health award P30CA47904; grants from the Pediatric Low-Grade Glioma Initiative via the National Brain Tumor Society and the Ellie Kavalieros Fund of the Children's Hospital of Pittsburgh Foundation; and the Pediatric Clinical and Translational Research Center, supported by the National Institutes of Health through grants UL1 RR024153 and ULITR000005. The imaging postprocessing was supported by the lan's (Ian Yagoda) Friends Foundation grant, of 9-10 months. ${ }^{2}$ Despite multiple clinical trials, irradiation is the only therapy that is of proved clinical benefit. Cancer peptide vaccines work by administering epitopes from antigens that are overexpressed in tumor cells to trigger the patient's immune response. The results of a pilot clinical trial targeting 3 gliomaassociated antigens, interleukin-13 receptor $\alpha 2$, EphA2, and survivin, in children with newly diagnosed malignant brain stem

the Society of Pediatric Radiology Pilot Award, and a National Library of Medicine grant 5T15LM007059-27.

Paper previously presented at: Annual Meeting of the American Society of Neuroradiology and the Foundation of the ASNR Symposium, May 18-23, 2013; San Diego, California.

Please address correspondence to Ashok Panigrahy, MD, Department of Pediatric Radiology, Children's Hospital of Pittsburgh of UPMC, 4401 Penn Ave, Pittsburgh, PA 15224; e-mail: panigrahya@upmc.edu

- Indicates open access to non-subscribers at www.ajnr.org

三 Indicates article with supplemental on-line appendix and table.

Indicates article with supplemental on-line photos.

http://dx.doi.org/10.3174/ajnr.A4428 
gliomas have been recently published. ${ }^{3}$ Pseudoprogression was observed in parallel to immunologic responses. ${ }^{3}$ Pseudoprogression, defined radiologically as a transient increase in the size of contrast-enhancing tumor on structural MR imaging, is thought to result from local tissue inflammation due to vasogenic edema and abnormal vessel permeability. Thus, assessment of pseudoprogression in peptide-based immunotherapy of pediatric brain stem gliomas presents a challenge for clinical management. Pseudoprogression can currently only be determined retrospectively after a period during which treatment is potentially halted, thus creating the evident problem of stopping treatment at a time when it is possibly at its highest efficacy.

Because structural MR imaging cannot reliably differentiate inflammation from recurrent tumor, ${ }^{4}$ advanced neuroimaging techniques such as DWI have been evaluated to assess brain tumor therapeutic response, ${ }^{5-12}$ including discriminating between pseudoprogression and true progression. We evaluated DWI as a potential tool to discriminate tumor response and true tumor progression, by using information available close to the time of suspected progression. The apparent diffusion coefficient is a quantitative measure reflecting the observed net movement of water calculated from DWI and has been shown to correlate with tissue cellularity in tumors, ${ }^{5-12}$ likely due to restriction of extracellular water motion in tightly packed tumor cells. Modulation of diffusion measurements has been previously observed in both preclinical and human studies of immunotherapy. ${ }^{13-15}$ While mean tissue ADC may be a useful measure for distinguishing tumor from other masses, it may be problematic for quantifying change with time. Opposing heterogeneous responses (ie, different areas of tumor with increasing and decreasing diffusion) may neutralize each other with no change in overall mean ADC. ${ }^{16}$ To overcome this limitation, we applied parametric response mapping (PRM), ${ }^{6,17-19}$ formerly known as functional diffusion mapping, to evaluate the response to immunotherapy with time by quantifying voxelwise changes in ADC. ${ }^{20}$ Our study differs from prior studies by evaluating immunotherapy, by its exclusive focus on pediatric brain tumors, and by using information from $>2$ time points in PRM. We hypothesized that serial PRMs calculated at multiple time points during immunotherapy could differentiate pseudoprogression from true progression in pediatric brain stem gliomas.

\section{MATERIALS AND METHODS}

\section{Demographics, Clinical Characteristics, and Study Design}

The cohort consisted of the 21 children (9 males) with DIPG enrolled in our institutional glioma vaccine trial (ClinicalTrials.gov No. NCT01130077). ${ }^{3}$ Before enrollment, patients had completed 5000- to 6000-cGy involved field fractionated radiation therapy, with or without concurrent chemotherapy; postirradiation chemotherapy precluded enrollment. Adequate organ function, absolute lymphocyte count of $\geq 500$, performance status of $\geq 60$, and human leukocyte antigen-A2 + status were required for vaccine treatment initiation. Patients with increased edema or mass effect after irradiation did not start the vaccination until this had resolved (up to 12 weeks after completion of irradiation).

Signed institutional review board-approved informed consent was required for human leukocyte antigen screening and initiation of therapy. Patients received subcutaneous injections of glioma-associated antigen-derived human leukocyte antigen$\mathrm{A}^{\star} 0201$-restricted peptides and a tetanus toxoid peptide (TetA830) emulsified in Montanide ISA-51 (Seppic, Puteaux, France) and concurrent intramuscular injections of $30 \mu \mathrm{g} / \mathrm{kg}$ of the toll-like receptor ligand, Hiltonol (poly-ICLC; Oncovir, Washington, DC), every 3 weeks $\times 8$ followed by a maintenance phase, every 6 weeks. ${ }^{3}$

Participants were evaluated with neurologic examinations and laboratory testing as previously described. ${ }^{3} \mathrm{MR}$ imaging (including DWI) was performed before initiating vaccine therapy (week 0 ) and at weeks $6,15,21$, after initiating vaccine therapy, and 12 -week intervals thereafter. More frequent scans were obtained if clinically warranted. Between May 2009 and October 2012, we enrolled 21 newly diagnosed patients: 15 with DIPG treated with irradiation alone and 6 with DIPG treated with irradiation and concurrent chemotherapy (On-line Table). Patients received $2-11$ doses of vaccine (median, 7 ). The mean age at diagnosis was $9 \pm 4.0$ years, and median survival was 56.3 weeks (range, $6.4-$ 24.9 months).

\section{Pseudoprogression}

Because the development of pseudoprogression is an area of concern in immunotherapy studies, the trial incorporated detailed guidelines for managing possible pseudoprogression. ${ }^{3}$ If tumor enlargement or increased enhancement or both were noted on structural MR imaging and the patient was neurologically worse, sufficient to warrant initiation of corticosteroid administration or an increase in corticosteroid dose, subsequent doses of vaccine and poly-ICLC were withheld. Imaging and clinical assessments were performed at 4-week intervals thereafter, until it was determined whether the clinical and imaging changes reflected pseudoprogression or true progression. If the subject improved clinically on declining corticosteroid doses that could be weaned to $\leq 0.1-\mathrm{mg} / \mathrm{kg} /$ day dexamethasone for $\geq 1$ week and the MR imaging changes improved or resolved, the patient was presumed to have had pseudoprogression and could restart vaccine treatment with $67 \%$ of the poly-ICLC dose (ie, $20 \mu \mathrm{g} / \mathrm{kg}$ ). Conversely, if the repeat $\mathrm{MR}$ imaging findings were unchanged or worse and the patient's clinical status had not improved despite increased corticosteroid doses, the patient was removed from the study due to presumed true tumor progression.

\section{MR Imaging and Diffusion-Weighted Imaging}

Imaging was conducted on 1.5T MR imaging systems with most of the studies performed on a 1.5T HDx system (GE Healthcare, Milwaukee, Wisconsin). Diffusion-weighted images were acquired at section thicknesses of 4-5 mm (1-mm gap), a TR ranging from 6000 to $8000 \mathrm{~ms}$, and $b=0$ and $1000 \mathrm{~s} / \mathrm{mm}^{2}$ per clinical protocol. To register every time point onto a common space for subsequent analysis, we chose the earliest time point available with volumetric imaging (T1- or T2-weighted, which in most cases was a 3D T2-Cube; GE Healthcare) after completion of radiation therapy and before vaccine therapy as a structural reference. A minimum of 3 diffusion imaging time points (preor post-initial vaccine) were required for analysis. Gradient- 


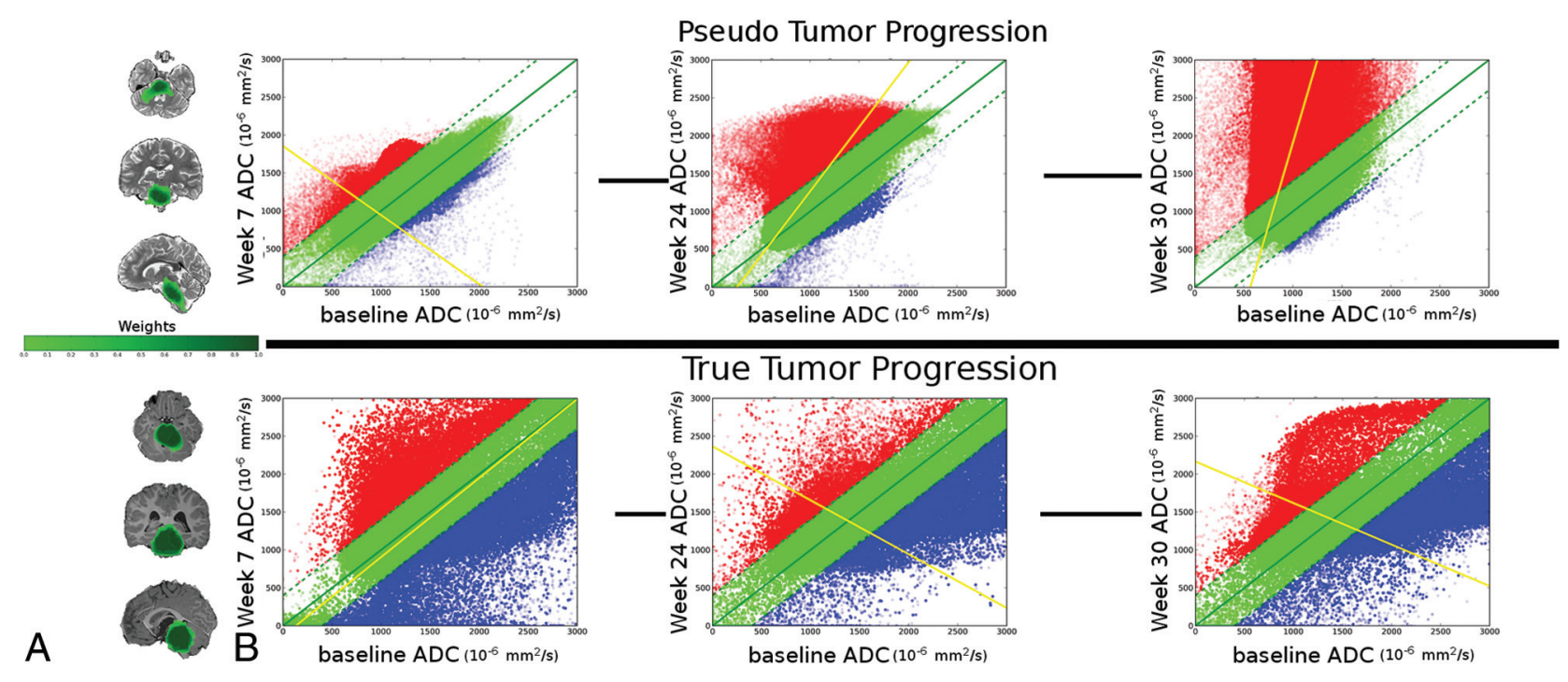

FIG 1. A, Tumor ROI for a patient with confirmed pseudoprogression (top) and a patient with true tumor progression without pseudoprogression (bottom). The color scale indicates the proportion of scans in which each voxel was classified as tumor tissue (voxel weights). B, Sample serial PRM maps at weeks 7, 24, and 30 compared with the baseline scan before vaccine therapy. Plots show coregistered voxels at baseline compared with the indicated time point. Green voxels indicate no significant change above or below the predefined threshold of $\pm 0.4 \mathrm{~mm}{ }^{2} / \mathrm{ms}$. Red voxels show a significant increase in ADC, and blue voxels, a decrease in ADC with time. Point opacity is proportional to the voxel weight (ie, how much does the voxel contribute to the PRM metric calculation in the weighted model).

echo or susceptibility-weighted imaging was also available for assessment of blood products.

\section{Image Registration}

A semiautomated processing pipeline ${ }^{21}$ was developed by using Nipype (http://nipy.sourceforge.net/nipype). ${ }^{22}$ Tumor area was manually delineated for each patient at each time point. We excluded regions with blood product that might cause EPI distortion. Tumor volume delineation was consistently performed for every patient in diffusion space, by using the B0 and ADC images to avoid any bias related to variations in institutional protocol. FLAIR imaging was also used to confirm the margin of the tumor. We performed a 2-step registration method to minimize potential registration errors: First the diffusion-weighted images were brain extracted and preregistered to a medium-resolution T2-weighted image acquired at the same time point by using a 6- $d$ frigid-body registration. The medium-resolution images were then registered to the chosen high-resolution structural image, and the resulting registration matrix was applied to the preregistered diffusion images. Linear registration was performed with FLIRT (FMRIB Linear Image Registration Tool; http://www. fmrib.ox.ac.uk/ $)^{23}$ by using an affine registration algorithm, followed by affine Fourier registration with Analysis of Functional Neuro Images software (ANFI; http://afni.nimh.nih. gov/afni/). ${ }^{24}$ We generated an aggregate-tumor ROI by adding all registered delineated tumor areas in common space and only including regions present in at least $10 \%$ of all time points.

\section{Serial PRM Postprocessing}

Parametric response maps were generated by an operator blinded to pseudoprogression diagnosis for every time point in reference to the baseline scan by calculating the voxelwise difference in the apparent diffusion coefficient within the aggregate tumor ROI
Pseudo Tumor Progression
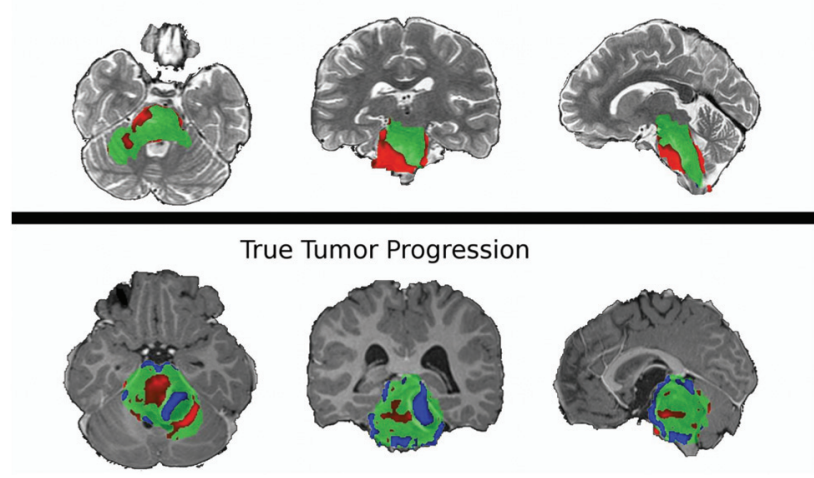

FIG 2. Sample PRM snapshots for a patient with confirmed pseudoprogression (top) and a patient with true tumor progression without pseudoprogression (bottom) give a spatiotemporal reference to tumor characterization. ADC maps are coregistered onto a common space, and voxelwise subtraction is calculated between each subsequent time point and the baseline scan. Green voxels indicate no significant change above or below the predefined threshold of \pm 0.4 $\mathrm{mm}^{2} / \mathrm{s}$. Red voxels show a significant increase in ADC, and blue voxels, a decrease in ADC compared with the baseline. There is evidence of spatial heterogeneity of diffusion within the brain stem tumor of both patients.

(Figs 1 and 2). We chose a significant change threshold of \pm 0.4 $\mathrm{mm}^{2} / \mathrm{s}$, as empirically determined by Ellingson et $\mathrm{al},{ }^{6}$ to represent the $95 \%$ confidence interval of temporal ADC variation in normal brain tissue. Patient-level summary measures were created following generation of PRMs. Fractional increased ADC (fiADC) measured by the percentage of voxels above this threshold reflects a decrease in cellularity and potentially indicates an inflammatory response or necrotic tissue. ${ }^{19,25}$ Fractional decreased ADC (fdADC) reflects the relative degree of hypercellularity and potentially indicates true tumor progression. We also looked at the ratio of fiADC to fdADC (PRMratio), calculated as fiADC / (fdADC + $0.01)^{26}$ (to account for instances in which $\mathrm{fdADC}=0$ ), as a po- 
tential marker for the overall trend of tissue progression. Wholetumor volume and mean ADC were also extracted from the same ROI as that used for PRM analysis.

\section{Statistical Analysis}

At the time of analysis, all patients in this study were deceased and can therefore be presumed to have had true tumor progression as an overall disease outcome. We therefore categorized patients without confirmed pseudoprogression as experiencing true progression (in contrast to patients whose eventual tumor progression was preceded by pseudoprogression). We tested for average differences in fiADC, fdADC, and PRMratio values between these 2 groups, with log-transformation of each to limit the influence of large values. The analysis was restricted to PRM values measured from scans performed no later than 12 weeks following a subject's final vaccination, because later scans are relevant only for retrospective assessment of pseudoprogression. Associations between each measure and pseudoprogression status were modeled by using weighted generalized estimating equations (WGEE) with independence working correlation. Because patients with rapid disease progression undergo fewer scans, the cluster size (number of scans per person) is informative. Regression analysis used inverse weighting by cluster size and an adjusted inference to "typical" PRM results for each patient rather than the full set of PRM results (which would implicitly contain information about prognosis). ${ }^{25,27}$ Statistical analyses were performed by using SAS/STAT statistical software, Version 9.4 (SAS Institute, Cary, North Carolina) and R, Version 3.0.1 (http://www.r-project.org/).

\section{RESULTS}

\section{Pseudoprogression Cases and Overall Survival Time}

Four children (19\%) developed acute neurologic worsening associated with increased tumor size and/or enhancement several months after beginning the vaccination, with subsequent clinical and radiographic improvement on corticosteroids, consistent with pseudoprogression (On-line Fig 1). One of these patients experienced a prolonged objective response, maintained until 19.5 months postdiagnosis as previously reported. ${ }^{3}$ Median survival from the time of diagnosis among patients with DIPG with pseudoprogression was 19.1 months, compared with 12.5 months in those without pseudoprogression.

\section{Differences in Serial PRM Metrics and Standard Measures (Mean ADC and Tumor Volume) between Patients with Pseudoprogression and True Progression}

A total of $151 \mathrm{MR}$ imaging time points for 21 patients had ADC maps available, with 3 scans excluded due to image-registration failure and an additional 2, due to artifacts in the diffusion imaging. Although the patients were on a treatment trial with scheduled follow-up, imaging time points varied due to scheduling windows and use of DWI. Serial PRM metrics for each patient are shown in Fig 3, with colored lines connecting PRM results for each subject's nonbaseline time points. The 3 rows display 3 PRM metrics (fiADC, fdADC, and the fiADC/fdADC or
PRMratio) as the $y$-axis for each plot. The $\mathrm{x}$-axis for each plot is the number of weeks after the first vaccine treatment (in contrast to the time from diagnosis, which is used for survival analysis). The 3 columns are panels created for ease of presentation, with patients sorted by increasing survival time. Each patient appears in all 3 rows, but in only 1 column. There is 1 pseudoprogression case in the second column and 3 in the third column (dashed lines), reflecting the longer survival for patients with pseudoprogression. Figure 3 suggests that patients with pseudoprogression (dashed lines) had a higher fiADC and higher PRMratio compared with patients whose vaccine therapy was halted due to true progression (solid lines) for most postbaseline scans. These trends are examined further in Fig 4, which shows the same PRM metrics for the same patients (with the same color coding across both Figs 3 and 4) for pseudoprogression and true progression but without information about scan timing.

To compare PRM metrics assessed earlier than the current (retrospective) standards for pseudoprogression, Fig 4 shows only the serial diffusion data used for statistical modeling, which is limited to time points from baseline until 12 weeks after the vaccine was halted (96 scans; $2-8$ scans per patient; median, 4 scans per patient). Assessed by using WGEE models accounting for informative clustering, patients with pseudoprogression, on average, had higher log-transformed PRMratios $(P=.01)$ and fiADCs $(P=.0004)$ and no statistically significant difference for lower log-transformed fdADCs $(P=.12)$ than patients without pseudoprogression. The fitted average fiADC/fdADC or PRMratio for a scan compared with baseline was 0.4 for patients without pseudoprogression (95\% CI, 0.3-0.6) and 3.7 for patients with pseudoprogression (95\% CI, 0.8-18.0).

There was no significant difference in mean ADC or tumor volume between patients with pseudoprogression and true progression. In the 4 patients with pseudoprogression, the greatest percentage change in mean $\mathrm{ADC}$ from the baseline measurement ranged from a $24 \%$ decrease to an $86 \%$ increase (median, $33 \%$ increase), compared with $-25 \%$ to $36 \%$ (median, $5 \%$ ) for the 17 remaining patients. Raw data for change in both mean ADC and tumor volume are found in the On-line Table. Using the same timeframe (postvaccine scans until 12 weeks after the last vaccine dose) and the same analysis approach (WGEE), we explored the association between mean ADC and tumor volume with pseudoprogression, controlling for baseline values. Neither mean ADC $(P=.55)$ nor tumor volume $(P=.44)$ was associated with pseudoprogression.

For a post hoc sensitivity analysis, we examined PRM, comparing baseline measures only to the time points most proximal to decisions about continuing vaccine therapy when progression (or pseudoprogression) was suspected. We defined these time points as up to 6 weeks before the last vaccine and up to 3 weeks after the last vaccine (allowing for no missed vaccines if pseudoprogression was identified). Twenty-seven scans with DWI met these criteria, 1-2 scans in 18 patients. Again the magnitude and direction of effects for fiADC and fdADC were maintained (and statistical significance strengthened) comparing pseudoprogression versus true progression; however, 2 of the 4 patients with pseudoprogression did not have scans within this timeframe. Other explor- 


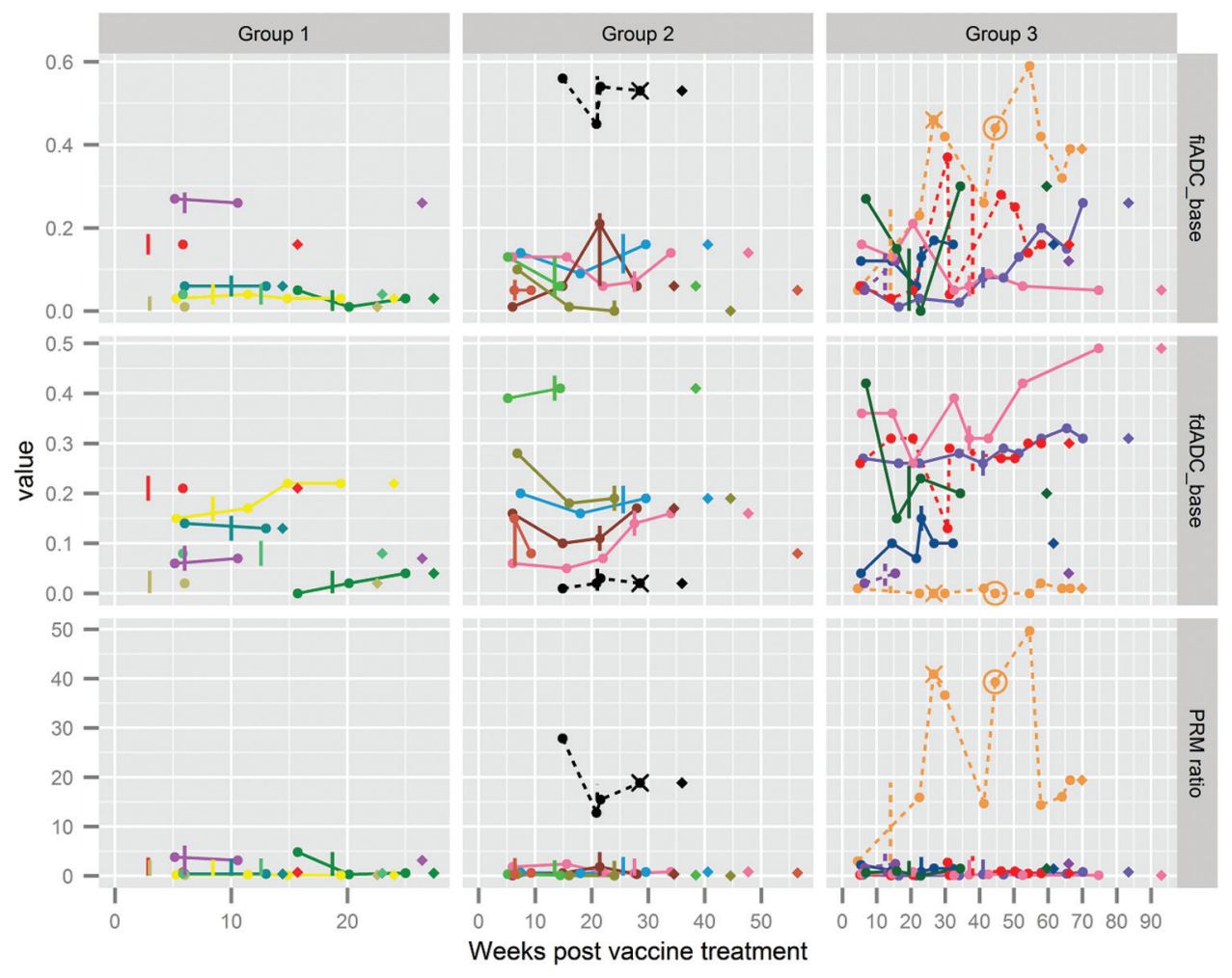

FIG 3. Serial PRM metric and disease trajectories for 21 pediatric patients with brain stem gliomas. Although the patients were on a treatment trial with scheduled follow-up, imaging time points varied due to scheduling windows and use of DWI. Serial PRM metrics for each patient are shown, with colored lines connecting PRM results for each subject's nonbaseline time points. Columns divide patients into groups by increasing overall survival from the start of vaccine therapy (14-27 weeks, 28-56 weeks, 57-93 weeks). Rows display fractional increased ADC, fractional decreased ADC, and PRMratio compared with the baseline (prevaccine) scan. Each PRM measurement is indicated by a circle, connected by solid lines for patients without pseudoprogression and dashed lines for patients with eventual diagnosis of pseudoprogression. Vertical lines indicate the date of the last vaccine for each patient. For 2 patients with psuedoprogression, vaccine treatment was restarted (date shown as X) 8 and 13 weeks after the initial halt. One of these patients underwent a second treatment stoppage (date shown as a circle). If one examined the time from the last vaccine dose (vertical line or circle for the patient who restarted therapy) to death ( $\diamond)$, patients survived 4-56 weeks after halting vaccine therapy.

atory analyses examined the robustness of our results to select tumor ROIs and exclude patients treated with bevacizumab (Online Appendix and On-line Fig 2).

\section{DISCUSSION}

This study, to our knowledge, is the first demonstration of the use of serial diffusion-weighted imaging and parametric response mapping in distinguishing pseudoprogression and true progression in immunotherapy trials for pediatric brain tumors. Childhood brain stem tumors treated with peptide-based vaccination had a notable rate of pseudoprogression, all with transient increases in tumor size or enhancement, with new or worsening neurologic deficits, and subsequent clinical improvement and MR imaging stabilization or improvement after administration of corticosteroids and suspension of vaccine therapy. ${ }^{3}$ Accurately identifying and managing such patients are essential to avoid both premature termination of therapy and unacceptable neurologic decline, a particular concern in children with DIPG who may develop significant neurologic deterioration with changes in mass effect. Our results suggest that PRM used to characterize temporal diffusion profiles is better able to distinguish pseudoprogression from true progression than mean ADC measurement or tumor volume. This outcome is likely related to treatment-related heterogeneity, in which there may be a mix of viable tumor (low ADC), necrosis (high ADC), and vasogenic edema (high ADC) within the tumor ROI. When measuring change in the mean ADC value of the tumor ROI, the potentially lower ADC values of active tumor foci likely blended with the higher ADC values found in areas of edema and necrosis. ${ }^{9}$

While these results are promising clinically, further study is required for greater understanding of the mechanisms underlying advanced MR imaging manifestations of immunotherapy-induced inflammatory response in the brain. ${ }^{14,28,29}$ One hypothesis is that immunotherapy effects on the tumor microenvironment lead to transient vasodilation, increased vessel permeability, and local inflammation, with a resultant increase in contrast enhancement and edema that mimics early tumor progression. An increase in ADC may correspond with tissue hypocellularity due to either treatment-related inflammation and/or tumor reduction. ${ }^{30}$ Animal models have demonstrated fractional increase in ADC by PRM as early as 24 hours following introduction of 1,3bis(2-chloroethyl)-1-nitrosourea ${ }^{15}$ and a detectable increase in ADC within 2 days following interleukin-13 receptor $\alpha 2$ T-cell injection. ${ }^{13}$ Progressive separation observed in long-term diffusion profiles between some patients with confirmed pseudoprogression versus true tumor progression in our study supports the use of parametric response maps as supplementary imaging biomarkers for monitoring tumor response in the setting of 

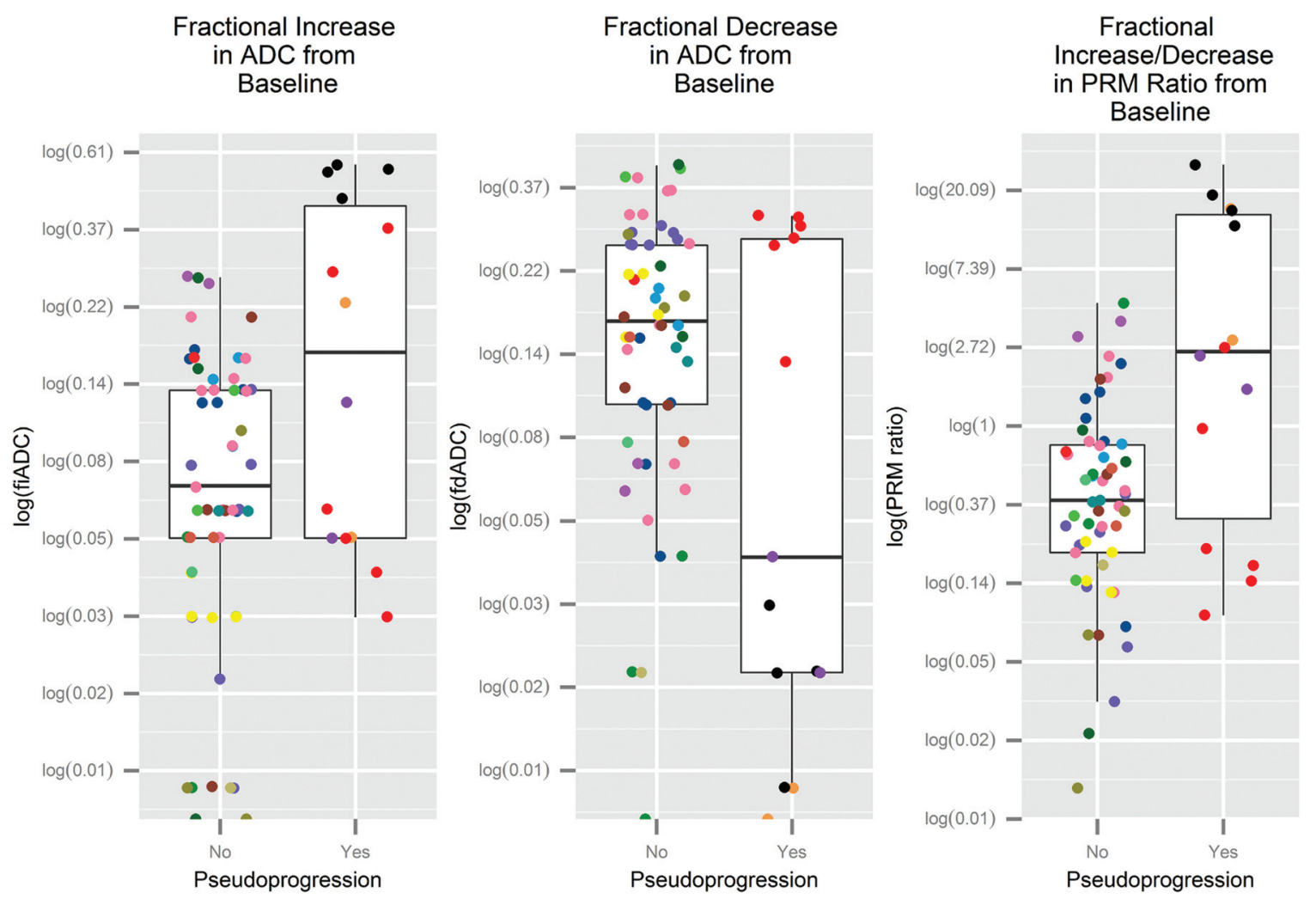

Note: Restricted to values collected within 12 weeks of last vaccine.

FIG 4. Boxplots of log-fractional increased ADC [log(fiADC)], log-fractional decreased ADC [log(fdADC)], and log-ratio of fiADC/fdADC [log (PRMratio)]. Values are obtained from PRMs from 75 postbaseline scans no more than 12 weeks after the last vaccine date, each compared with the patient's baseline scan. Cohorts are confirmed pseudoprogression ( $n=4$ patients) and true tumor progression (no pseudoprogression, $n=$ 17 patients). Data points of the same color are the same patient's PRM metrics for multiple scans, each compared with the baseline. Figure 3 uses the same coloring scheme (but includes time points $>12$ weeks after last vaccine date).

peptide-based immunotherapy. In particular, fiADC and the PRMratio appear to be the strongest candidates as potential early biomarkers for determining pseudoprogression.

PRMs have been shown to predict treatment response and survival in the setting of adult glioblastoma multiforme. ${ }^{17,18}$ However, these studies used only 2 imaging time points, in contrast to our study, in which multiple PRMs were analyzed per patient. Some limitations of the use of PRM in adult tumors are related to poor image registration of lesions resulting from changes in mass effect and tumor contour, which was less of an issue in our pediatric brain stem gliomas. Given that PRM measurements can be confounded by normal tissue, ${ }^{31}$ we used weighted-PRM techniques to control for changes in tumor size from each time point, without substantive changes in study results.

Study limitations include a small number of pseudoprogression cases and lack of biopsy confirmation for pseudoprogression. (Biopsy is a high-risk procedure for DIPG lesions.) The peak period of pseudoprogression following radiation therapy for DIPG is generally within the first 3 months. ${ }^{3}$ Anything after that time is presumed to be true tumor progression, unless the patient has received vaccine-related immunotherapy, as in our study. The peak period of pseudoprogression related to radiation therapy had likely passed before initiation of vaccine therapy, and the observed cases of pseudoprogression occurred after several doses of vaccine. Furthermore, patients with increased edema or mass effect after irradiation did not start vaccination until this had re- solved (up to 12 weeks after completion of irradiation). Regardless, the objective of our study was not to elucidate mechanisms for pseudoprogression by using PRM but to explore its utility in the characterization of pseudoprogression in pediatric DIPG.

\section{CONCLUSIONS}

Our study is the first to suggest that serial PRM may be useful to identify pseudoprogression in children with DIPG receiving peptide-based immunotherapy. The valuable properties of diffusion imaging with PRM analysis as an in vivo imaging biomarker include its translatability to the clinical arena, its quantitative nature, and its ease of use and cost effectiveness. The accurate identification of pseudoprogression versus true tumor progression is crucial in determining the optimal management of this novel treatment. We have 3 strong candidates (fiADC, fdADC, and PRMratio) for the development of a predictive model of pseudoprogression, in conjunction with other types of biomarkers that may assist in the treatment of children undergoing immunotherapy. We believe that combining diffusion imaging metrics with clinical information and standard MR imaging will allow timely discrimination of pseudoprogression and true progression, enabling optimal use of immunotherapy. Our preliminary observations, which analyzed 96 scans in 21 patients, should be validated in a planned multi-institutional clinical trial before being used to guide clinical management. 


\section{ACKNOWLEDGMENTS}

We thank University of Pittsburgh Cancer Institute Clinical Research Services for regulatory management; Andres Salazar, Oncovir, for provision of poly-ICLC; physicians who referred their patients; and the patients and families who participated in this trial. We also thank Angela K. Connelly, Sharon Dibridge, Fern Wasco, and Melanie Gieraltowski for research coordination.

Disclosures: Hideho Okada—RELATED: Grant: National Institutes of Health*; UNRELATED: Patents (planned, pending, or issued): Stemline Therapeutics, ${ }^{*}$ Comments: The peptides used for vaccines in the current study are my invention (with other coinventors) and have been exclusively licensed to Stemline Therapeutics for further development; Royalties: Stemline Therapeutics, ${ }^{\star}$ Comments: The same as above. Stemline pays royalties to the University of Pittsburgh; OTHER: Hideho Okada is an inventor in the US Patent Application No. 60,611, 797 (Utility Patent Application) "Identification of An IL-13 Receptor Alpha2 Peptide Analogue Capable of Enhancing Stimulation of Glioma-Specific CTL Response." An exclusive licensing agreement has been completed on this application between University of Pittsburgh and Stemline. Due to the potential conflicts of interest, Hideho Okada did not solely interpret any data in the current study. ${ }^{*}$ Money paid to the institution.

\section{REFERENCES}

1. Poussaint TY, Rossi A. Imaging of pediatric brain and spinal cord tumors. In: Hodler J, Schulthess GK. Diseases of the Brain, Head \& Neck, Spine 2012-2015: Diagnostic Imaging and Interventional Techniques. New York: Springer-Verlag; 2012:203-14

2. Jennings MT, Freeman ML, Murray MJ. Strategies in the treatment of diffuse pontine gliomas: the therapeutic role of hyperfractionated radiotherapy and chemotherapy. J Neurooncol 1996;28:207-22 Medline

3. Pollack IF, Jakacki RI, Butterfield LH, et al. Antigen-specific immune responses and clinical outcome after vaccination with glioma-associated antigen peptides and polyinosinic-polycytidylic acid stabilized by lysine and carboxymethylcellulose in children with newly diagnosed malignant brainstem and nonbrainstem gliomas. J Clin Oncol 2014;32:2050-58 CrossRef Medline

4. Hygino da Cruz LC Jr, Rodriguez I, Domingues RC, et al. Pseudoprogression and pseudoresponse: imaging challenges in the assessment of posttreatment glioma. AJNR Am J Neuroradiol 2011;32: 1978-85 CrossRef Medline

5. Bode MK, Ruohonen J, Nieminen MT, et al. Potential of diffusion imaging in brain tumors: a review. Acta Radiol 2006;47:585-94 CrossRef Medline

6. Ellingson BM, Malkin MG, Rand SD, et al. Validation of functional diffusion maps (fDMs) as a biomarker for human glioma cellularity. J Magn Reson Imaging 2010;31:538-48 CrossRef Medline

7. Sugahara T, Korogi Y, Kochi M, et al. Usefulness of diffusion-weighted MRI with echo-planar technique in the evaluation of cellularity in gliomas. J Magn Reson Imaging 1999;9:53-60 CrossRef Medline

8. Lyng H, Haraldseth O, Rofstad EK. Measurement of cell density and necrotic fraction in human melanoma xenografts by diffusion weighted magnetic resonance imaging. Magn Reson Med 2000;43: 828-36 CrossRef Medline

9. Chenevert TL, Stegman LD, Taylor JM, et al. Diffusion magnetic resonance imaging: an early surrogate marker of therapeutic efficacy in brain tumors. J Natl Cancer Inst 2000;92:2029-36 CrossRef Medline

10. Guo AC, Cummings TJ, Dash RC, et al. Lymphomas and high-grade astrocytomas: comparison of water diffusibility and histologic characteristics. Radiology 2002;224:177-83 CrossRef Medline

11. Hayashida Y, Hirai T, Morishita S, et al. Diffusion-weighted imaging of metastatic brain tumors: comparison with histologic type and tumor cellularity. AJNR Am J Neuroradiol 2006;27:1419-25 Medline

12. Kinoshita $\mathrm{M}$, Hashimoto $\mathrm{N}$, Goto $\mathrm{T}$, et al. Fractional anisotropy and tumor cell density of the tumor core show positive correlation in diffusion tensor magnetic resonance imaging of malignant brain tumors. Neuroimage 2008;43:29-35 CrossRef Medline

13. Lazovic J, Jensen MC, Ferkassian E, et al. Imaging immune response in vivo: cytolytic action of genetically altered $\mathrm{T}$ cells directed to glioblastoma multiforme imaging immune response in vivo: cytolytic action of genetically altered $\mathrm{T}$ cells directed to glioblastoma multiforme. Clin Cancer Res 2008;14:3832-39 CrossRef Medline

14. Vrabec M, Van Cauter S, Himmelreich U, et al. MR perfusion and diffusion imaging in the follow-up of recurrent glioblastoma treated with dendritic cell immunotherapy: a pilot study. Neuroradiology 2011;53:721-31 CrossRef Medline

15. Moffat B, Chenevert TL, Meyer CR, et al. The functional diffusion map: an imaging biomarker for the early prediction of cancer treatment outcome. Neoplasia 2006;8:259-67 CrossRef Medline

16. LaViolette PS, Mickevicius NJ, Cochran EJ, et al. Precise ex vivo histological validation of heightened cellularity and diffusion-restricted necrosis in regions of dark apparent diffusion coefficient in 7 cases of high-grade glioma. Neuro Oncol 2014;16:1599-606 CrossRef Medline

17. Hamstra DA, Chenevert TL, Moffat BA, et al. Evaluation of the functional diffusion map as an early biomarker of time-to-progression and overall survival in high-grade glioma. Proc Natl Acad Sci U S A 2005;102:16759-64 CrossRef Medline

18. Moffat BA, Chenevert TL, Lawrence TS, et al. Functional diffusion map: a noninvasive MRI biomarker for early stratification of clinical brain tumor response. Proc Natl Acad Sci US A 2005;102:5524-29 CrossRef Medline

19. Ellingson BM, Cloughesy TF, Zaw T, et al. Functional diffusion maps (fDMs) evaluated before and after radiochemotherapy predict progression-free and overall survival in newly diagnosed glioblastoma. Neuro Oncol 2012;14:333-43 CrossRef Medline

20. Ellingson BM, Malkin MG, Rand SD, et al. Volumetric analysis of functional diffusion maps is a predictive imaging biomarker for cytotoxic and anti-angiogenic treatments in malignant gliomas. J Neurooncol 2011;102:95-103 CrossRef Medline

21. Ceschin R, Panigrahy A, Gopalakrishnan V. sfDM: open-source software for temporal analysis and visualization of brain tumor diffusion MR using serial functional diffusion mapping. Cancer Inform 2015;14(suppl 2):1-9 CrossRef Medline

22. Gorgolewski K, Burns CD, Madison C, et al. Nipype: a flexible, lightweight and extensible neuroimaging data processing framework in python. Front Neuroinform 2011;5:13 CrossRef Medline

23. Jenkinson M, Beckmann CF, Behrens TE, et al. FSL. Neuroimage 2012;62:782-90 CrossRef Medline

24. Cox RW. AFNI: software for analysis and visualization of functional magnetic resonance neuroimages. Comput Biomed Res 1996; 29:162-73 CrossRef Medline

25. Pavlou M. Analysis of clustered data when the cluster size is informative. University College London, 2012. http://discovery.ucl. ac.uk/1357842/1/mpavlou_thesis.pdf.

26. Rodriguez Gutierrez D, Manita M, Jaspan T, et al. Serial MR diffusion to predict treatment response in high-grade pediatric brain tumors: a comparison of regional and voxel-based diffusion change metrics. Neuro Oncol 2013;15:981-89 CrossRef Medline

27. Williamson JM, Datta S, Satten G. Marginal analyses of clustered data when cluster size is informative. Biometrics 2003;59:36-42 CrossRef Medline

28. Yang I, Huh NG, Smith ZA, et al. Distinguishing glioma recurrence from treatment effect after radiochemotherapy and immunotherapy. Neurosurg Clin N Am 2010;21:181-86 CrossRef Medline

29. Okada H, Pollack IF. Do we need novel radiologic response criteria for brain tumor immunotherapy? Expert Rev Neurother 2011;11: 619-22 CrossRef Medline

30. Wieduwilt MJ, Valles F, Issa S, et al. Immunochemotherapy with intensive consolidation for primary CNS lymphoma: a pilot study and prognostic assessment by diffusion-weighted MRI. Clin Cancer Res 2012;18:1146-55 CrossRef Medline

31. Grech-Sollars M, Saunders DE, Phipps KP, et al. Challenges for the functional diffusion map in pediatric brain tumors. Neuro Oncol 2014;16:449-56 CrossRef Medline 